\title{
Statistical mechanics of transport processes of fluids under confined conditions
}

\author{
V. Rudyak, A. Belkin \\ Novosibirsk State University of Architecture and Civil Engineering, Novosibirsk, Russia \\ valery.rudyak@mail.ru, a_belkin@ngs.ru
}

PACS 47.61.-k, 47.10.-g

DOI 10.17586/2220-8054-2015-6-3-366-377

The problem of adequately describing transport processes of fluids in confined conditions is solved using methods of nonequilibrium statistical mechanics. The 'fluid-channel wall' system is regarded as a two-fluid medium, in which each phase has a particular velocity and temperature. The obtained results show that the transport equations in confined spaces should contain not only the stress tensor and the heat flux vector, but also the interfacial forces responsible for the transfer of momentum and heat due to the interaction with the wall surfaces. The stress tensor and the heat flux vector fluid can be expressed in terms of the effective viscosity and thermal conductivity. However, the constitutive relations contain additive terms that correspond to fluid-surface interactions. Thus, not only do the fluid transport coefficients in nanochannels differ from the bulk transport coefficients, but they are also not only determined by the parameters of the fluid.

Keywords: Micro flow, transport coefficients, nonequilibrium statistical mechanics, confined system.

Received: 16 January 2015

\section{Introduction}

The extensive study of liquid and gas microflows over the last two decades has been motivated by the emergence of a large number of microfluidics tools and systems. These flows are currently use in biochemistry, medicine, pharmacology, biology, thermal engineering, catalysis, etc. An important aspect of the active use of various micro and nanoflows is the development of a new generation of resource-saving technologies with low power consumption. The efficiency of micro and nanodevices is largely determined by the transport processes occurring in the fluid flows. Thus, for example, mixing in micromixers is due to diffusion processes, and the energy spent for fluid circulation depends on the fluid viscosity. Today, however, it is known that transport processes in confined geometries are significantly different from those in the bulk. Diffusion, for example, is anisotropic [1,2], fluid viscosity is differed from the value in the bulk [3-5] and the fluid in nanochannel has a nonhomogeneous structure [6,7].

Difficulties in measuring transport coefficients in small channels or pores are obvious. In fact, at present, there are no reliable experimental data. However, already today, we can argue with certainty, first, that the fluid viscosity in small channels is inhomogeneous over the channel cross-section and, second, that its average value is significantly different from the value in bulk. This, in particular, is suggested by the results of molecular dynamics simulations $[2,7]$. Layers of structured fluid with characteristic scales on the order of nanometers are formed near the channel walls. In such channels, fluctuations in the number of molecules, momentum, and energy become important [8].

Existing experimental data on the pressure drop are very contradictory. Some authors argue that the pressure drop is described by the classical equations of fluid dynamics (see, e.g., [3]). However, there are indications that the pressure drop coefficient is much higher than the classical value (e.g., [3]) and less than it [9]. Often, these discrepancies are explained 
by the slip boundary conditions on the micro or nanochannel walls (see for example [10] and referenced therein). However, in our opinion, the situation is more delicate. Transport processes are processes of relaxation of large-scale fluctuations, such as gradients of observed macroscopic variables: density, velocity, temperature, etc. Fluctuations can be maintained, in particular, by external forces with respect to the system. Transport processes in rarefied gases are easily interpreted: they are due to transfer of the corresponding microscopic characteristics on scales of the order of the mean free path of the molecules. In liquids, transport processes are much more complicated. For example, the viscosity is due not only to the momentum transfer in collisions of molecules, but also to the destruction of short-range order and diffusion processes of momentum transfer in the system. In the vicinity of the surface, an important factor in the equalization of momentum and energy in flow is the interaction of fluid molecules with the surface's molecules. In a nanochannel with a characteristic cross-sectional area on the order of $5 \mathrm{~nm}$, almost half of all interactions of fluid molecules are their collisions with the molecules of the channel walls. Under such conditions, it becomes meaningless to speak about the viscosity of the fluid separately. The viscosity of the fluid becomes a property of the entire 'fluid-nanochannel wall' system. Of course, it is not easy to study this 'viscosity' experimentally. However, to develop an appropriate apparatus, it is first necessary to construct an adequate theory of transport processes, which should be used in interpreting experimental data. The aim of this work is to develop such a theory. It is constructed from the first principles using methods of nonequilibrium statistical mechanics.

\section{The transport equations of the system}

Since the states of the molecules of the fluid and the channel walls are significantly different (in particular, the walls are at rest and the fluid moves), the system is a peculiar twofluid medium. The properties of this medium are described in this paper using the apparatus that we developed previously to describe dispersed media [11-14]. In this case, the 'fluid-channel wall' system is treated as a two-fluid medium, each of which phases consists of the same type of molecules and is characterized by their macroscopic variables: density, velocity, and temperature. The dynamics of the system is described by the N-particle distribution function $F_{N}$ which satisfies the Liouville equation:

$$
\partial F_{N} / \partial t+L_{N} F_{N}=0
$$

in which the Liouville operator is defined as:

$$
L_{N}=\sum_{\alpha, \varphi=1}^{2} \sum_{i=1}^{N_{\alpha}}\left[\frac{\mathbf{p}_{i}}{m_{\alpha}} \cdot \frac{\partial}{\partial \mathbf{r}_{i}}+\frac{1}{2} \sum_{j=1}^{N_{\varphi}} \mathbf{F}_{i j} \cdot\left(\frac{\partial}{\partial \mathbf{p}_{i}}-\frac{\partial}{\partial \mathbf{p}_{j}}\right)\right] .
$$

Here $\mathbf{r}_{i}, m_{i}$, and $\mathbf{p}_{i}$ are the coordinate of the center of mass, mass, and momentum of the $i$-th molecule of phase $\alpha$.

We will characterize the state of the system by partial values of the density $n_{\alpha}$, momentum $\mathbf{p}_{\alpha}$, and energy $E_{\alpha}$. These quantities are the averages of the corresponding dynamical variables:

$$
\hat{n}_{\alpha}(\mathbf{r})=\sum_{i=1}^{N_{\alpha}} \delta\left(\mathbf{r}_{i}-\mathbf{r}\right), \quad \hat{\mathbf{p}}_{\alpha}(\mathbf{r})=\sum_{i=1}^{N_{\alpha}} \mathbf{p}_{i} \delta\left(\mathbf{r}_{i}-\mathbf{r}\right), \quad \hat{E}_{\alpha}\left(\mathbf{r}_{i}-\mathbf{r}\right)=\sum_{i=1}^{N_{\alpha}} E_{i} \delta\left(\mathbf{r}_{i}-\mathbf{r}\right),
$$


where $E_{i}$ is the energy of the $i$-th molecule. Applying the Liouville operator to the dynamic densities (2), we obtain the following transport equation for them:

$$
\dot{\hat{n}}_{\alpha}=-\nabla \cdot \hat{\mathbf{J}}_{1 \alpha}, \quad \dot{\hat{\mathbf{p}}}_{\alpha}=-\nabla \cdot \hat{\mathbf{J}}_{2 \alpha}+\hat{\mathbf{j}}_{2 \alpha}, \quad \dot{\hat{E}}_{\alpha}=-\nabla \cdot \hat{\mathbf{J}}_{3 \alpha}+\hat{j}_{3 \alpha}
$$

The operators of the number flux of molecules $\hat{\mathbf{J}}_{1 \alpha}$, momentum flux $\hat{\mathbf{J}}_{2 \alpha}$, the energy flux $\hat{\mathbf{J}}_{3 \alpha}$, and the interfacial force operators $\hat{\mathbf{j}}_{2 \alpha}$ and $\hat{j}_{3 \alpha}$ are defined as follows:

$$
\begin{gathered}
\hat{\mathbf{J}}_{1 \alpha}=\sum_{i=1}^{N_{\alpha}} \frac{\mathbf{p}_{i}}{m_{\alpha}} \delta\left(\mathbf{r}_{i}-\mathbf{r}\right), \quad \hat{\mathbf{J}}_{2 \alpha}=\sum_{i=1}^{N_{\alpha}} \frac{\mathbf{p}_{i} \mathbf{p}_{i}}{m_{\alpha}} \delta\left(\mathbf{r}_{i}-\mathbf{r}\right)+\frac{1}{2} \sum_{i=1}^{N_{\alpha}} \sum_{\varphi}^{f, b} \sum_{j=1}^{N_{\varphi}} \mathbf{r}_{i j} \mathbf{F}_{i j} \int_{0}^{1} d \eta \delta\left(\mathbf{r}_{j}-\mathbf{r}+\eta \mathbf{r}_{i j}\right), \\
\hat{\mathbf{j}}_{2 \alpha}=\frac{1}{2} \sum_{i=1}^{N_{\alpha}} \sum_{j=1}^{N_{\varphi \neq \alpha}} \mathbf{F}_{i j}\left[\delta\left(\mathbf{r}_{i}-\mathbf{r}\right)+\delta\left(\mathbf{r}_{j}-\mathbf{r}\right)\right] \\
\hat{\mathbf{J}}_{3 \alpha}=\sum_{i=1}^{N_{\alpha}} \frac{\mathbf{p}_{i} E_{i}}{m_{\alpha}} \delta\left(\mathbf{r}_{i}-\mathbf{r}\right)+\frac{1}{4} \sum_{i=1}^{N_{\alpha}} \sum_{\varphi=1}^{f, b} \sum_{j=1}^{N_{\varphi}}\left(\frac{\mathbf{p}_{i}}{m_{\alpha}}+\frac{\mathbf{p}_{j}}{m_{\varphi}}\right) \cdot \mathbf{r}_{i j} \mathbf{F}_{i j} \int_{0}^{1} d \eta \delta\left(\mathbf{r}_{j}-\mathbf{r}+\eta \mathbf{r}_{i j}\right), \\
\hat{j}_{3 \alpha}=\frac{1}{4} \sum_{i=1}^{N_{\alpha}} \sum_{j=1}^{N_{\varphi \neq \alpha}} \mathbf{F}_{i j} \cdot\left(\frac{\mathbf{p}_{i}}{m_{\alpha}}+\frac{\mathbf{p}_{j}}{m_{\varphi}}\right)\left[\delta\left(\mathbf{r}_{i}-\mathbf{r}\right)+\delta\left(\mathbf{r}_{j}-\mathbf{r}\right)\right] .
\end{gathered}
$$

The hydrodynamic velocities of the fluid (hereinafter, we use the subscript $f$ ) and the walls (subscript $b$ ) are defined as follows: $\mathbf{u}_{f}(\mathbf{r}, t)=\mathbf{p}_{f}(\mathbf{r}, t) / m_{f} n_{f}, \mathbf{u}_{b}(\mathbf{r}, t)=0$. Transport equations of the hydrodynamic variables can be obtained by averaging the density transport equations (3) over the ensemble $F_{N}$ and using a locally accompanying coordinate system for the fluid which moves relative to the laboratory system with velocity $\mathbf{u}_{f}$. It can be shown that these equations have the following form:

$$
\begin{gathered}
\frac{\partial n_{f}}{\partial t}+\nabla \cdot\left(n_{f} \mathbf{u}_{f}\right)=0, \quad \frac{\partial n_{b}}{\partial t}=0 \\
\rho_{f} \frac{\partial \mathbf{u}_{f}}{\partial t}+\rho_{f} \mathbf{u}_{f} \cdot \nabla \mathbf{u}_{f}=-\nabla \cdot \mathbf{J}_{2 f}^{\prime}+\mathbf{j}_{2 f}, \quad-\nabla \cdot \mathbf{J}_{2 b}^{\prime}+\mathbf{j}_{2 b}=0 \\
\frac{\partial E_{f}^{\prime}}{\partial t}+\nabla \cdot\left(\mathbf{u}_{f} E_{f}^{\prime}\right)=-\nabla \cdot \mathbf{J}_{3 f}^{\prime}-\left(\mathbf{J}_{2 f}^{\prime}+\mathbf{J}_{f b}\right): \nabla \mathbf{u}_{f}-\mathbf{j}_{f b} \cdot \mathbf{u}_{f}+j_{3 f}^{\prime} \\
\frac{\partial E_{b}^{\prime}}{\partial t}=-\nabla \cdot \mathbf{J}_{3 b}^{\prime}-j_{3 f}^{\prime}+\mathbf{J}_{f b}: \nabla \mathbf{u}_{f} .
\end{gathered}
$$

Here $\mathbf{A}=\langle\hat{\mathbf{A}}\rangle$, the angle brackets denote an average over the ensemble $F_{N}$, and all primed quantities were obtained from the corresponding unprimed ones by the momentum transformation: $\mathbf{p}_{i}^{\prime}=\mathbf{p}_{i}-m_{\alpha} \mathbf{u}_{\alpha}$. Moreover, additional microscopic densities are introduced:

$$
\hat{\mathbf{J}}_{f b}=-\frac{1}{4} \sum_{i=1}^{N_{f}} \sum_{j=1}^{N_{b}} \mathbf{r}_{i j} \mathbf{F}_{i j} \int_{0}^{1} d \eta \delta\left(\mathbf{r}_{j}-\mathbf{r}+\eta \mathbf{r}_{i j}\right), \quad \hat{\mathbf{j}}_{f b}=\frac{1}{2} \sum_{i=1}^{N_{f}} \sum_{j=1}^{N_{b}} \mathbf{F}_{i j} \delta\left(\mathbf{r}_{i}-\mathbf{r}\right) .
$$




\section{Nonequilibrium distribution function}

The transport equations (4) are not closed; they contain the fluxes and interphases forces which should be connected with macroscopic variables. To calculate the fluxes $\mathbf{J}_{\alpha i}^{\prime}$ and forces $\mathbf{j}_{\alpha i}^{\prime}$ we need to determine the distribution function $F_{N}$. Thus the problem of deriving the transport equations and constitutive relations reduces to finding a solution of equation (1) for the macroscopic level of the system description. Due to the linearity of equation (1), its solution can be sought in the form of the sum of the quasi-equilibrium distribution function $F_{N 0}$ and the dissipation function $F_{N 1}$ :

$$
F_{N}=F_{N 0}+F_{N 1}
$$

The function $F_{N 0}$ is obtained from the extremum condition for the information entropy $S=-k\left\langle\ln F_{N 0}\right\rangle$ ( $k$ is Boltzmann's constant) for the given average values of the number of particles, momentum, and energy of the particles of each medium (fluid and channel walls) (see $[11,15])$. The distribution function obtained in this way corresponds to the two-fluid description of the system. In the derivation of $F_{N 0}$, it should only be taken into account that the macroscopic velocity of the wall is equal to zero and that the temperatures of the walls and the fluid can be different. Thus the quasi-equilibrium distribution function has the following form:

$$
\begin{gathered}
F_{N 0}=Q_{0}^{-1} \exp \left\{-\int d \mathbf{r}\left[\beta_{f}(\mathbf{r}, t) \hat{E}_{f}^{\prime}(\mathbf{r})-\nu_{f}(\mathbf{r}, t) \hat{n}_{f}(\mathbf{r})+\beta_{b}(\mathbf{r}, t) \hat{E}_{b}(\mathbf{r})-\nu_{b}(\mathbf{r}, t) \hat{n}_{b}(\mathbf{r})\right]\right\}, \\
Q_{0}=\left\langle\exp \left\{-\sum_{\alpha=f}^{b} \sum_{k=1}^{3} \int g_{k \alpha}(\mathbf{r}, t) \hat{G}_{k \alpha}(\mathbf{r})\right\}\right\rangle, \\
\hat{G}_{1 \alpha}(\mathbf{r})=\hat{n}_{\alpha}(\mathbf{r}), \quad \hat{G}_{2 \alpha}(\mathbf{r})=\hat{\mathbf{p}}_{\alpha}(\mathbf{r}), \quad \hat{G}_{3 \alpha}(\mathbf{r})=\hat{E}_{\alpha}(\mathbf{r}) .
\end{gathered}
$$

Here $g_{3 \alpha}=\beta_{\alpha}=1 / k T_{\alpha}$ is the local inverse temperature of the component $\alpha, \nu_{\alpha}=-\beta_{\alpha} \mu_{\alpha}$, $g_{1 \alpha}=\beta_{\alpha}\left(-\mu_{\alpha}+m_{\alpha} \mathbf{u}_{\alpha}^{2} / 2\right), g_{2 \alpha}=-\beta_{\alpha} \mathbf{u}_{\alpha}$, and $\mu_{\alpha}$ is the local chemical potential. The average values of the particle number density of the component and its energy calculated for the quasiequilibrium ensemble (6) are:

$$
\left\langle\hat{n}_{\alpha}(\mathbf{r})\right\rangle_{0}=\frac{\delta \ln Q_{0}}{\delta \nu_{\alpha}(\mathbf{r})}, \quad\left\langle\hat{E}_{\alpha}^{\prime}(\mathbf{r})\right\rangle_{0}=\frac{\delta \ln Q_{0}}{\delta \beta_{\alpha}(\mathbf{r})} .
$$

Here, averaging over the ensemble (6) is denoted by the subscript 0 in the angle brackets. For the thermodynamics of the system to be defined by the function $F_{N 0}$, it is necessary that the macroscopic variables coincide with their quasi-equilibrium values: $n_{\alpha}(\mathbf{r}, t)=\left\langle\hat{n}_{\alpha}(\mathbf{r})\right\rangle_{0}$, $E_{f}^{\prime}(\mathbf{r}, t)=\left\langle\hat{E}_{f}^{\prime}(\mathbf{r})\right\rangle_{0}$.

Because $F_{N 0}$ is an even function of the momenta $\mathbf{p}_{i}^{\prime}$ and the coordinates $\mathbf{r}_{i j}$, the mean values of the nondiagonal elements of the stress tensor and the tensor $\mathbf{J}_{f b}$ calculated from it are equal to zero:

$$
\mathbf{J}_{2 f}^{\prime 0}(\mathbf{r}, t)=\frac{1}{3}\left\langle\hat{\mathbf{J}}_{2 f}^{\prime}(\mathbf{r}): \mathbf{U}\right\rangle_{0}=p_{f}(\mathbf{r}, t) \mathbf{U}, \quad \mathbf{J}_{f b}^{0}(\mathbf{r}, t)=\frac{1}{3}\left\langle\hat{\mathbf{J}}_{f b}(\mathbf{r}): \mathbf{U}\right\rangle_{0}=p_{f b}(\mathbf{r}, t) \mathbf{U} .
$$

Here, $p_{f}$ is the partial pressure of the fluid, which we assume to be isotropic and $\mathbf{U}$ is the unit tensor of second rank. For the same reason, the other fluxes and interfacial forces are equal to zero, and the equations of multifluid hydrodynamics for the quasi-equilibrium ensemble (6) are the Euler-type equations: 


$$
\begin{gathered}
\frac{\partial n_{f}}{\partial t}+\nabla \cdot\left(n_{f} \mathbf{u}_{f}\right)=0, \quad \rho_{f} \frac{\partial \mathbf{u}_{f}}{\partial t}+\rho_{f} \mathbf{u}_{f} \cdot \nabla \mathbf{u}_{f}=-\nabla p_{f}, \\
\frac{\partial E_{f}^{\prime}}{\partial t}+\mathbf{u}_{f} \nabla \cdot E_{f}^{\prime}=-\left(E_{f}^{\prime}+p_{f}+p_{f b}\right) \nabla \cdot \mathbf{u}_{f}, \quad \frac{\partial E_{b}^{\prime}}{\partial t}=p_{f b} \nabla \cdot \mathbf{u}_{f} .
\end{gathered}
$$

It is evident that these equations derived for the ensemble $F_{N 0}$ do not describe dissipative processes in the system. To construct the nonequilibrium distribution function, it is necessary to solve the linear inhomogeneous equation (see Eq. (5)):

$$
\partial F_{N 1} / \partial t+L_{N} F_{N 1}=-\left(\partial F_{N 0} / \partial t+L_{N} F_{N 0}\right)
$$

Applying the Liouville operator to the function $F_{N 0}$, we obtain:

$$
\begin{aligned}
& L_{N} F_{N 0}=-F_{N 0}\left(t_{0}\right) \int d \mathbf{r}\left[\hat{\mathbf{J}}_{1 f} \cdot \nabla \nu_{f}-\beta_{f}\left(\hat{\mathbf{J}}_{2 f}-m_{f} \mathbf{u}_{f} \hat{\mathbf{J}}_{1 f}\right): \nabla \mathbf{u}_{f}+\right. \\
&\left(\hat{\mathbf{J}}_{3 f}-\hat{\mathbf{J}}_{2 f} \cdot \mathbf{u}_{f}+m_{f} \frac{\mathbf{u}_{f}^{2}}{2} \hat{\mathbf{J}}_{1 f}\right) \cdot \nabla \beta_{f}+ \\
&\left.\hat{\mathbf{J}}_{1 b} \cdot \nabla \nu_{b}+\hat{\mathbf{J}}_{3 b} \cdot \nabla \beta_{b}-\hat{\mathbf{j}}_{2 f} \cdot \beta_{f} \nabla \mathbf{u}_{f}+\hat{j}_{3 f}\left(\beta_{f}-\beta_{b}\right)\right] .
\end{aligned}
$$

The right side of equation (8) contains also time derivatives of hydrodynamic quantities which are determined from the transport equations (7) using the complete distribution function. The time derivative of the quasi-equilibrium distribution function can be written as follows:

$$
\begin{gathered}
\partial F_{N 0} / \partial t=T F_{N 0}+T F_{N 1}, \\
T F_{N j}=F_{N 0} \sum_{\alpha=f}^{b} \sum_{k=1}^{3} \int d \mathbf{r} \Delta \tilde{G}_{k \alpha}(\mathbf{r}) \frac{\partial_{j} \phi_{k \alpha}(\mathbf{r}, t)}{\partial t}, \quad j=0,1, \quad \phi_{1 \alpha}=\nu_{\alpha}, \quad \phi_{2 \alpha}=\mathbf{u}_{\alpha}, \quad \phi_{3 \alpha}=-\beta_{\alpha}, \\
\Delta \tilde{G}_{k \alpha}=\hat{\tilde{G}}_{k \alpha}-\tilde{G}_{k \alpha}, \quad \hat{\tilde{G}}_{1 \alpha}=\hat{G}_{1 \alpha}, \quad \hat{\tilde{G}}_{2 \alpha}=\beta_{\alpha} \hat{G}_{2 \alpha}^{\prime}, \quad \hat{\tilde{G}}_{3 \alpha}=\hat{G}_{3 \alpha}^{\prime}, \\
\frac{\partial_{j} \phi_{k \alpha}(\mathbf{r}, t)}{\partial t}=\left\langle\frac{\partial \phi_{k \alpha}(\mathbf{r}, t)}{\partial t}\right\rangle_{j} .
\end{gathered}
$$

Then Eq. (8) becomes:

$$
\partial F_{N 1} / \partial t+\left(T+L_{N}\right) F_{N 1}=-\left(T+L_{N}\right) F_{N 0}
$$

A method for solving this equation was developed and described in detail previously [11-14]. Omitting cumbersome calculations, we give the explicit form of the nonequilibrium distribution function obtained using this method for the system considered:

$$
\begin{gathered}
F_{N 1}(t)=\pi\left(t, t_{0}\right) S_{\left(t-t_{0}\right)}^{(N)} F_{N 1}\left(t_{0}\right)+\sum_{\alpha}^{f, b} \sum_{k=1}^{5} \int_{t_{0}}^{t} d t_{1} \int d \mathbf{r} \int d \mathbf{r}^{\prime} \pi\left(t, t_{1}\right) S_{\left(t-t_{1}\right)}^{(N)} F_{N 0}\left(t_{1}\right) \Delta \mathbf{I}_{k \alpha} \cdot \mathbf{Y}_{k \alpha}\left(\mathbf{r}^{\prime}, t_{1}\right) \\
\Delta \mathbf{I}_{k \alpha}=\hat{\mathbf{I}}_{k \alpha}\left(\mathbf{r}, \mathbf{r}^{\prime}\right)-\mathbf{I}_{k \alpha}^{0}\left(\mathbf{r}, \mathbf{r}^{\prime}\right), \quad \mathbf{I}_{i a}^{0}=\left\langle\hat{\mathbf{I}}_{i a}\right\rangle_{0}
\end{gathered}
$$

Here $S_{\left(t-t_{0}\right)}^{(N)}$ is the displacement operator along the trajectory of $N$ particles. The operator $\pi\left(t, t_{1}\right)$ has the form of an infinite series in the thermodynamic forces $[11,16]$ : 


$$
\begin{gathered}
\pi\left(t, t_{1}\right)=\sum_{n=0}^{\infty} \pi^{n}\left(t, t_{1}\right), \quad \pi^{0}\left(t, t_{1}\right)=1, \\
\pi^{n}\left(t, t_{1}\right)=(-1)^{n} \int_{t_{1}}^{t} d t_{2} \ldots \int_{t_{1}}^{t} d t_{n+1} \prod_{i=2}^{n+1} S_{-\left(t-t_{i}\right)}^{(N)} T\left(t_{i}\right) S_{\left(t-t_{i}\right)}^{(N)},
\end{gathered}
$$

where the product is formed starting from terms with the largest $i$. For weakly nonequilibrium systems (in ordinary hydrodynamics, this corresponds to the Navier-Stokes approximation), this series can be truncated to the first term. In this case the operator $\pi\left(t, t_{1}\right)$ is equal to unity. The fluxes $\hat{\mathbf{I}}_{k \alpha}$ and thermodynamic forces $\hat{\mathbf{Y}}_{k \alpha}$ are given by:

$$
\begin{aligned}
& \hat{\mathbf{I}}_{1 f}=\hat{\mathbf{J}}_{1 f}^{\prime}(\mathbf{r})\left(\delta\left(\mathbf{r}-\mathbf{r}^{\prime}\right)-\frac{\beta_{f}(\mathbf{r})}{n_{f}(\mathbf{r})}\left(\frac{\delta p_{f}(\mathbf{r})}{\delta \nu_{f}\left(\mathbf{r}^{\prime}\right)}\right)_{\beta_{f}}\right) \\
& \hat{\mathbf{I}}_{1 b}=\hat{\mathbf{J}}_{1 b}^{\prime}(\mathbf{r}) \delta\left(\mathbf{r}-\mathbf{r}^{\prime}\right), \quad \mathbf{Y}_{1 \alpha}=-\nabla^{\prime} \nu_{\alpha} \\
& \hat{\mathbf{I}}_{2 f}=\left(\hat{\mathbf{J}}_{2 f}^{\prime}(\mathbf{r})+\hat{\mathbf{J}}_{f b}(\mathbf{r})\right) \beta_{f} \delta\left(\mathbf{r}-\mathbf{r}^{\prime}\right)-\left[E_{f}^{\prime}(\mathbf{r})+p_{f}(\mathbf{r})+p_{f b}(\mathbf{r})\right] \times \\
& {\left[\hat{n}_{f}(\mathbf{r})\left(\frac{\delta \nu_{f}(\mathbf{r})}{\delta E_{f}^{\prime}\left(\mathbf{r}^{\prime}\right)}\right)_{n_{f}}-\hat{E}_{f}^{\prime}(\mathbf{r})\left(\frac{\delta \beta_{f}(\mathbf{r})}{\delta E_{f}^{\prime}\left(\mathbf{r}^{\prime}\right)}\right)_{n_{f}}\right] \mathbf{U}-} \\
& n_{f}(\mathbf{r})\left[\hat{n}_{f}(\mathbf{r})\left(\frac{\delta \nu_{f}(\mathbf{r})}{\delta n_{f}\left(\mathbf{r}^{\prime}\right)}\right)_{E_{f}^{\prime}}-\hat{E}_{f}^{\prime}(\mathbf{r})\left(\frac{\delta \beta_{f}(\mathbf{r})}{\delta n_{f}\left(\mathbf{r}^{\prime}\right)}\right)_{E_{f}^{\prime}}\right] \mathbf{U} \\
& \hat{\mathbf{I}}_{2 b}=p_{f b}(\mathbf{r})\left[\hat{n}_{b}(\mathbf{r})\left(\frac{\delta \nu_{b}(\mathbf{r})}{\delta E_{b}^{\prime}\left(\mathbf{r}^{\prime}\right)}\right)_{n_{b}}-\hat{E}_{b}^{\prime}(\mathbf{r})\left(\frac{\delta \beta_{b}(\mathbf{r})}{\delta E_{b}^{\prime}\left(\mathbf{r}^{\prime}\right)}\right)_{n_{b}}\right] \mathbf{U}, \quad \mathbf{Y}_{2 \alpha}=-\nabla^{\prime} \cdot \mathbf{u}_{f}, \\
& \hat{\mathbf{I}}_{3 f}=\hat{\mathbf{J}}_{3 f}^{\prime}(\mathbf{r}) \delta\left(\mathbf{r}-\mathbf{r}^{\prime}\right)+\hat{\mathbf{p}}_{f}^{\prime}(\mathbf{r}) \frac{\beta_{f}(\mathbf{r})}{m_{f} n_{f}(\mathbf{r})}\left(\frac{\delta p_{f}(\mathbf{r})}{\delta \beta_{f}\left(\mathbf{r}^{\prime}\right)}\right)_{\nu_{f}} \\
& \hat{\mathbf{I}}_{3 b}=\hat{\mathbf{J}}_{3 b}^{\prime}(\mathbf{r}) \delta\left(\mathbf{r}-\mathbf{r}^{\prime}\right), \quad \mathbf{Y}_{3 \alpha}=-\nabla^{\prime} \beta_{\alpha}, \\
& \hat{\mathbf{I}}_{4 f}=-\hat{\mathbf{j}}_{2 f}(\mathbf{r}) \beta_{f} \delta\left(\mathbf{r}-\mathbf{r}^{\prime}\right), \quad \mathbf{Y}_{4 f}=\mathbf{u}_{f}, \quad \mathbf{Y}_{4 b}=0, \\
& \hat{I}_{5 f}=\left(\hat{j}_{3 f}^{\prime}(\mathbf{r})+\frac{1}{2} \hat{\mathbf{j}}_{2 f}(\mathbf{r}) \cdot \mathbf{u}_{f}\right) \delta\left(\mathbf{r}-\mathbf{r}^{\prime}\right), \quad Y_{5 f}=\beta_{f}-\beta_{b}, \quad \mathbf{Y}_{5 b}=0 .
\end{aligned}
$$

In these expressions, the subscript at a bracket indicates the expression in the bracket is calculated for the fixed variables specified in the subscript, $\nabla^{\prime}=\partial / \partial \mathbf{r}^{\prime}$.

The fluxes $\hat{\mathbf{I}}_{k \alpha}$ are generally nonlocal. The characteristic scales of its nonlocality are about the size of the internal structural elements of the medium and the time of their interaction. For simple fluids, this type of nonlocality can be neglected. Therefore we can replace the functional derivatives with the partial derivatives, which will be done below. 


\section{Constitutive relations and transport coefficients}

Using the nonequilibrium distribution function (9), we obtain fluid transport equations in flows bounded by surfaces. The constitutive relations for the stress tensor, heat flux vector, and interfacial forces entering these equations are generally nonlocal and retarded:

$$
\begin{gathered}
\mathbf{f}_{i a}(\mathbf{r}, t)=\mathbf{f}_{i a}\left(\mathbf{r}, t_{0}\right)+\mathbf{f}_{i a}^{0}(\mathbf{r}, t)+\sum_{k=1}^{5} \sum_{\beta, \varphi}^{2} \int_{t_{0}}^{t} d t_{1} \int d \mathbf{r}^{\prime} \mathbf{M}_{i a}^{k \beta}\left(\mathbf{r}, \mathbf{r}^{\prime}, t-t_{1}\right) \cdot \mathbf{Y}_{k \beta}\left(\mathbf{r}^{\prime}, t_{1}\right), \\
\hat{\mathbf{f}}_{1 a}=\hat{\mathbf{J}}_{1 a}^{\prime}, \quad \hat{\mathbf{f}}_{2 a}=\hat{\mathbf{J}}_{2 a}^{\prime}, \quad \hat{\mathbf{f}}_{3 a}=\hat{\mathbf{J}}_{3 a}^{\prime}, \quad \hat{\mathbf{f}}_{4 a}=\hat{\mathbf{j}}_{2 a}, \\
\hat{f}_{5 a}=\hat{j}_{3 a}^{\prime}, \quad \hat{\mathbf{f}}_{6 f}=\hat{\mathbf{J}}_{f b}, \quad \hat{\mathbf{f}}_{7 f}=\hat{\mathbf{j}}_{f b}, \quad \hat{\mathbf{f}}_{6 b}=\hat{\mathbf{f}}_{7 b}=0 .
\end{gathered}
$$

The relaxation transport kernels included in these formulas are given by the relations:

$$
\mathbf{M}_{i a}^{k \beta}\left(\mathbf{r}, \mathbf{r}^{\prime}, t-t_{1}\right)=\left\langle\hat{\mathbf{f}}_{i a}(\mathbf{r}) S_{\left(t-t_{1}\right)}^{(N)} \Delta \mathbf{I}_{k \beta}\left(\mathbf{r}^{\prime}\right)\right\rangle_{0} .
$$

Given that the quasi-equilibrium distribution function is even in momenta and relative coordinates of the molecules, it can be shown that the uneven transport kernels (11) in these quantities are equal to zero. Moreover, in the constitutive relations (10), the stress tensor and the tensor $\mathbf{J}_{f b}$ can be split into a symmetric nondivergent part (denoted below by superscript $s$ ) and a divergent part (denoted by superscript 0 ). In this case, the constitutive relations for the fluid under confined conditions have the following form:

$$
\begin{aligned}
& \mathbf{J}_{2 f}^{\prime s}(\mathbf{r}, t)=\int_{t_{0}}^{t} d t_{1} \int d \mathbf{r}^{\prime}\left\langle\hat{\mathbf{J}}_{2 f}^{\prime s}(\mathbf{r}) S_{\left(t-t_{1}\right)}^{(N)} \Delta\left(\hat{\mathbf{J}}_{2 f}^{\prime s}\left(\mathbf{r}^{\prime}\right)+\hat{\mathbf{J}}_{f b}^{s}\left(\mathbf{r}^{\prime}\right)\right)\right\rangle_{0}: \beta_{f}\left(\mathbf{r}^{\prime}\right) \nabla^{\prime} \mathbf{u}_{f}\left(\mathbf{r}^{\prime}\right), \\
& \mathbf{J}_{i}^{0}(\mathbf{r}, t)=\int_{t_{0}}^{t} d t_{1} \int d \mathbf{r}^{\prime}\left\langle\hat{\mathbf{J}}_{i}^{0}(\mathbf{r}) S_{\left(t-t_{1}\right)}^{(N)} \Delta\left(\hat{\mathbf{I}}_{f}^{0}\left(\mathbf{r}^{\prime}\right)+\hat{\mathbf{I}}_{f b}^{0}\left(\mathbf{r}^{\prime}\right)\right)\right\rangle_{0}: \nabla^{\prime} \cdot \mathbf{u}_{f}\left(\mathbf{r}^{\prime}\right) \mathbf{U}, \\
& i=1,2, \quad \hat{\mathbf{J}}_{1}^{0}=\hat{\mathbf{J}}_{2 f}^{\prime 0}, \quad \hat{\mathbf{J}}_{2}^{0}=\hat{\mathbf{J}}_{f b}^{\prime 0}, \quad \hat{\mathbf{J}}_{i}^{0}=\left\langle\hat{\mathbf{J}}_{i}^{0}\right\rangle, \\
& \hat{\mathbf{I}}_{f}^{0}(\mathbf{r})=\hat{\mathbf{J}}_{2 f}^{\prime}(\mathbf{r}) \beta_{f}-\left(E_{f}^{\prime}(\mathbf{r})+p_{f}(\mathbf{r})\right) \mathbf{U} \times \\
& \left(\hat{n}_{f}(\mathbf{r})\left(\frac{\partial \nu_{f}}{\partial E_{f}^{\prime}}\right)_{n_{f}}-\hat{E}_{f}^{\prime}(\mathbf{r})\left(\frac{\partial \beta_{f}}{\partial E_{f}^{\prime}}\right)_{n_{f}}\right)-n_{f}(\mathbf{r})\left(\hat{n}_{f}(\mathbf{r})\left(\frac{\partial \nu_{f}}{\partial n_{f}}\right)_{E_{f}^{\prime}}-\hat{E}_{f}^{\prime}(\mathbf{r})\left(\frac{\partial \beta_{f}}{\partial n_{f}}\right)_{E_{f}^{\prime}}\right) \mathbf{U}, \\
& \hat{\mathbf{I}}_{f b}^{0}(\mathbf{r})=\hat{\mathbf{J}}_{f b}(\mathbf{r}) \beta_{f}-p_{f b}(\mathbf{r}) \mathbf{U} \times \\
& \left(\hat{n}_{f}(\mathbf{r})\left(\frac{\partial \nu_{f}}{\partial E_{f}^{\prime}}\right)_{n_{f}}-\hat{E}_{f}^{\prime}(\mathbf{r})\left(\frac{\partial \beta_{f}}{\partial E_{f}^{\prime}}\right)_{n_{f}}\right)+p_{f b}(\mathbf{r})\left(\hat{n}_{b}(\mathbf{r})\left(\frac{\partial \nu_{b}}{\partial E_{b}^{\prime}}\right)_{n_{b}}-\hat{E}_{b}^{\prime}(\mathbf{r})\left(\frac{\partial \beta_{b}}{\partial E_{b}^{\prime}}\right)_{n_{b}}\right) \mathbf{U}, \\
& \mathbf{j}_{2 f}(\mathbf{r}, t)=\frac{1}{2} \int_{t_{0}}^{t} d t_{1} \int d \mathbf{r}^{\prime}\left\langle\hat{\mathbf{j}}_{2 f}(\mathbf{r}) S_{\left(t-t_{1}\right)}^{(N)} \hat{\mathbf{j}}_{2 f}\left(\mathbf{r}^{\prime}\right)\right\rangle_{0}\left(\beta_{f}\left(\mathbf{r}^{\prime}\right)+\beta_{\mathbf{p}}\left(\mathbf{r}^{\prime}\right)\right) \mathbf{u}_{f}\left(\mathbf{r}^{\prime}\right) \\
& \mathbf{J}_{3 f}^{\prime}(\mathbf{r}, t)=-\int_{t_{0}}^{t} d t_{1} \int d \mathbf{r}^{\prime}\left\langle\hat{\mathbf{J}}_{3 f}^{\prime}(\mathbf{r}) S_{\left(t-t_{1}\right)}^{(N)} \Delta\left(\hat{\mathbf{J}}_{3 f}^{\prime}\left(\mathbf{r}^{\prime}\right)+\hat{\mathbf{p}}_{f}\left(\mathbf{r}^{\prime}\right) \frac{\beta_{f}\left(\mathbf{r}^{\prime}\right)}{m_{f} n_{f}\left(\mathbf{r}^{\prime}\right)}\left(\frac{\partial p_{f}}{\partial \beta_{f}}\right)_{\nu_{f}}\right)\right\rangle_{0} \cdot \nabla^{\prime} \beta_{f}\left(\mathbf{r}^{\prime}\right),
\end{aligned}
$$




$$
\begin{gathered}
j_{3 f}^{\prime}(\mathbf{r}, t)=\int_{t_{0}}^{t} d t_{1} \int d \mathbf{r}^{\prime}\left\langle\hat{j}_{3 f}^{\prime}(\mathbf{r}) S_{\left(t-t_{1}\right)}^{(N)} \Delta \hat{j}_{3 f}^{\prime}\left(\mathbf{r}^{\prime}\right)\right\rangle_{0}\left(\beta_{f}\left(\mathbf{r}^{\prime}\right)-\beta_{b}\left(\mathbf{r}^{\prime}\right)\right), \\
\mathbf{J}_{f b}^{s}(\mathbf{r}, t)=\int_{t_{0}}^{t} d t_{1} \int d \mathbf{r}^{\prime}\left\langle\hat{\mathbf{J}}_{f b}^{s}(\mathbf{r}) S_{\left(t-t_{1}\right)}^{(N)} \Delta\left(\hat{\mathbf{J}}_{2 f}^{\prime s}\left(\mathbf{r}^{\prime}\right)+\hat{\mathbf{J}}_{f b}^{s}\left(\mathbf{r}^{\prime}\right)\right)\right\rangle_{0}: \beta_{f}\left(\mathbf{r}^{\prime}\right) \nabla^{\prime} \mathbf{u}_{f}\left(\mathbf{r}^{\prime}\right), \\
\mathbf{j}_{f b}(\mathbf{r}, t)=\frac{1}{2} \int_{t_{0}}^{t} d t_{1} \int d \mathbf{r}^{\prime}\left\langle\hat{\mathbf{j}}_{f b}(\mathbf{r}) S_{\left(t-t_{1}\right)}^{(N)} \hat{\mathbf{j}}_{2 f}^{\prime}\left(\mathbf{r}^{\prime}\right)\right\rangle_{0} \cdot\left(\beta_{f}\left(\mathbf{r}^{\prime}\right)+\beta_{b}\left(\mathbf{r}^{\prime}\right)\right) \mathbf{u}_{f}\left(\mathbf{r}^{\prime}\right) .
\end{gathered}
$$

Equations (12) are nonlinear, nonlocal, and retarded constitutive relations. Its nonlocality is associated with the correlation between dissipative fluxes and thermodynamic forces and is due to the finite speed of propagation of disturbances in the medium. For weakly nonequilibrium systems for which the gradients of the observed macroscopic variables are relatively low (in classical fluid mechanics, this corresponds to Newton's constitutive equations for the stress tensor and Fourier's equations for the heat flux vector), this nonlocality and retardation can also be neglected. In addition, as noted above, the operator $\pi\left(t, t_{1}\right)=1$ in this case. If we neglect the initial values of the fluxes and interfacial forces, the constitutive equations in this case can be written as:

$$
\begin{gathered}
\mathbf{J}_{2 f}^{\prime}(\mathbf{r}, t)=p_{f}(\mathbf{r}, t) \mathbf{U}-\left(\boldsymbol{\mu}_{f f}^{s}+\boldsymbol{\mu}_{f b}^{s}\right): \nabla \mathbf{u}_{f}^{s}-\left(\boldsymbol{\mu}_{f f}^{0}+\boldsymbol{\mu}_{f b}^{0}\right): \nabla \cdot \mathbf{u}_{f} \mathbf{U}, \quad \mathbf{j}_{2 f}(\mathbf{r}, t)=-\boldsymbol{\nu} \cdot \mathbf{u}_{f} \\
\mathbf{J}_{3 f}^{\prime}(\mathbf{r}, t)=\boldsymbol{\lambda}_{f} \cdot \nabla \beta_{f}+\boldsymbol{\lambda}_{b} \cdot \nabla \beta_{b}, \quad j_{3 f}^{\prime}(\mathbf{r}, t)=\omega\left(\beta_{f}-\beta_{b}\right) \\
\mathbf{J}_{f b}(\mathbf{r}, t)=p_{f b}(\mathbf{r}, t) \mathbf{U}-\left(\boldsymbol{\mu}_{b f}^{s}+\boldsymbol{\mu}_{b b}^{s}\right): \nabla \mathbf{u}_{f}^{s}-\left(\boldsymbol{\mu}_{b f}^{0}+\boldsymbol{\mu}_{b b}^{0}\right): \nabla \cdot \mathbf{u}_{f} \mathbf{U} \\
\mathbf{j}_{f b}(\mathbf{r}, t)=-\boldsymbol{\sigma} \cdot \mathbf{u}_{f} .
\end{gathered}
$$

Here, transport coefficients $\boldsymbol{\mu}_{f f}^{s}, \boldsymbol{\mu}_{f f}^{0}, \boldsymbol{\mu}_{f b}^{0}$, and $\boldsymbol{\mu}_{f b}^{s}$ are fourth-rank tensors, $\boldsymbol{\nu}, \boldsymbol{\lambda}_{f}, \boldsymbol{\lambda}_{b}$ and $\boldsymbol{\sigma}$ are second-rank tensors, and $\varpi$ is a scalar. These quantities are given by the following relations:

$$
\begin{gathered}
\boldsymbol{\mu}_{i f}^{s}=\beta_{f} \int d t_{1} \int_{t_{0}}^{t} d \mathbf{r}^{\prime}\left\langle\hat{\mathbf{J}}_{i}^{\prime s}(\mathbf{r}) S_{\left(t-t_{1}\right)}^{(N)} \hat{\mathbf{J}}_{2 f}^{\prime s}\left(\mathbf{r}^{\prime}\right)\right\rangle_{0}, \quad i=f, b, \quad \hat{\mathbf{J}}_{f}^{\prime}=\hat{\mathbf{J}}_{2 f}^{\prime}, \quad \hat{\mathbf{J}}_{b}^{\prime}=\hat{\mathbf{J}}_{f b}^{\prime}, \\
\boldsymbol{\mu}_{i b}^{s}=\beta_{f} \int_{t_{0}}^{t} d t_{1} \int d \mathbf{r}^{\prime}\left\langle\hat{\mathbf{J}}_{i}^{\prime s}(\mathbf{r}) S_{\left(t-t_{1}\right)}^{(N)} \hat{\mathbf{J}}_{f b}^{s}\left(\mathbf{r}^{\prime}\right)\right\rangle_{0} \\
\boldsymbol{\mu}_{i f}^{0}=\int_{t_{0}}^{t} d t_{1} \int d \mathbf{r}^{\prime}\left\langle\hat{\mathbf{J}}_{i}^{\prime 0}(\mathbf{r}) S_{\left(t-t_{1}\right)}^{(N)} \hat{\mathbf{I}}_{f}^{0}\left(\mathbf{r}^{\prime}\right)\right\rangle_{0} \\
\boldsymbol{\mu}_{i b}^{0}=\int_{t_{0}}^{t} d t_{1} \int d \mathbf{r}^{\prime}\left\langle\hat{\mathbf{J}}_{i}^{\prime 0}(\mathbf{r}) S_{\left(t-t_{1}\right)}^{(N)} \hat{\mathbf{I}}_{f b}^{0}\left(\mathbf{r}^{\prime}\right)\right\rangle_{0}, \\
\boldsymbol{\lambda}_{f}=\int_{t_{0}}^{t} d t_{1} \int d \mathbf{r}^{\prime}\left\langle\hat{\mathbf{J}}_{3 f}^{\prime}(\mathbf{r}) S_{\left(t-t_{1}\right)}^{(N)} \Delta\left(\hat{\mathbf{J}}_{3 f}^{\prime}\left(\mathbf{r}^{\prime}\right)+\hat{\mathbf{p}}_{f}^{\prime}\left(\mathbf{r}^{\prime}\right) \frac{\beta_{f}\left(\mathbf{r}^{\prime}\right)}{m_{f} n_{f}\left(\mathbf{r}^{\prime}\right)}\left(\frac{\partial p_{f}}{\partial \beta_{f}}\right)_{\nu_{f}}\right)\right\rangle_{0}
\end{gathered}
$$




$$
\begin{gathered}
\boldsymbol{\lambda}_{b}=\int_{t_{0}}^{t} d t_{1} \int d \mathbf{r}^{\prime}\left\langle\hat{\mathbf{j}}_{3 f}^{\prime}(\mathbf{r}) S_{\left(t-t_{1}\right)}^{(N)} \Delta \hat{\mathbf{J}}_{3 b}^{\prime}\left(\mathbf{r}^{\prime}\right)\right\rangle_{0}, \\
\varpi=\int_{t_{0}}^{t} d t_{1} \int d \mathbf{r}^{\prime}\left\langle\hat{j}_{3 f}^{\prime}(\mathbf{r}) S_{\left(t-t_{1}\right)}^{(N)} \hat{j}_{3 f}^{\prime}\left(\mathbf{r}^{\prime}\right)\right\rangle_{0}, \\
\boldsymbol{\sigma}=\frac{1}{2}\left(\beta_{f}+\beta_{b}\right) \int_{t_{0}}^{t} d t_{1} \int d \mathbf{r}^{\prime}\left\langle\hat{\mathbf{j}}_{f b}(\mathbf{r}) S_{\left(t-t_{1}\right)}^{(N)} \hat{\mathbf{j}}_{2 f}\left(\mathbf{r}^{\prime}\right)\right\rangle_{0}, \\
\boldsymbol{\nu}=\frac{1}{2}\left(\beta_{f}+\beta_{b}\right) \int_{t_{0}}^{t} d t_{1} \int d \mathbf{r}^{\prime}\left\langle\hat{\mathbf{j}}_{2 f}(\mathbf{r}) S_{\left(t-t_{1}\right)}^{(N)} \hat{\mathbf{j}}_{2 f}\left(\mathbf{r}^{\prime}\right)\right\rangle_{0} .
\end{gathered}
$$

Note that in the case of incompressible channel walls, their hydrodynamic velocity equals zero, $\mathbf{u}_{b}=0$. The condition that the magnitudes of the interfacial forces $\mathbf{j}_{2 f}=\mathbf{j}_{2 b}$ and the momentum equation for the wall:

$$
-\nabla \cdot \mathbf{J}_{2 b}^{\prime}+\mathbf{j}_{2 b}=0
$$

imply that $\mathbf{j}_{2 b}^{\prime}=-\nabla \cdot \mathbf{J}_{2 b}^{\prime}$. If we neglect the bulk viscosity, the evolution equation of the fluid velocity can be written in simple form:

$$
\rho_{f} \frac{d \mathbf{u}_{f}}{d t}=-\nabla p_{f}-\nabla \cdot\left(\boldsymbol{\mu}_{f f}^{s}+\boldsymbol{\mu}_{f b}^{s}+\boldsymbol{\mu}_{b}^{s}\right): \nabla \mathbf{u}_{f}^{s}
$$

Thus, the viscosity of the fluid in confined geometry depends not only on the interaction between the fluid molecules (responsible tensor coefficient $\boldsymbol{\mu}_{f f}^{s}$ ). The effective viscosity contains the contributions due to the interaction with the surface, the coefficient $\boldsymbol{\mu}_{b}^{s}$ is given by the relation:

$$
\boldsymbol{\mu}_{b}^{s}(\mathbf{r})=\beta_{b} \int d \mathbf{r}^{\prime} \int_{t_{0}}^{t} d t_{1}\left\langle\hat{\mathbf{J}}_{2 b}^{\prime s}(\mathbf{r}) S_{\left(t-t_{1}\right)}^{(N)} \Delta \hat{\mathbf{J}}_{f b}^{s}\left(\mathbf{r}^{\prime}\right)\right\rangle_{0} .
$$

Further simplification of the constitutive relations is possible in systems having symmetrical properties. For an isotropic medium, the transport kernel (11) and the corresponding transport coefficients (14) are scalars multiplied by the isotropic tensors. Thus, for an isotropic medium, the local constitutive relations reduce to:

$$
\begin{gathered}
\mathbf{J}_{2 f}^{\prime}(\mathbf{r}, t)=p_{f} \mathbf{U}-\left(\mu_{f f}^{s}+\mu_{f b}^{s}+\mu_{b}^{s}\right) \nabla \mathbf{u}_{f}^{s}-\left(\mu_{f f}^{0}+\mu_{f b}^{0}+\mu_{b}^{0}\right) \nabla \cdot \mathbf{u}_{f} \mathbf{U}, \quad \mathbf{j}_{2 f}(\mathbf{r}, t)=0 \\
\mathbf{J}_{3 f}^{\prime}(\mathbf{r}, t)=\lambda_{f} \nabla \beta_{f}+\lambda_{b} \nabla \beta_{b}, \quad j_{3 f}^{\prime}(\mathbf{r}, t)=\omega\left(\beta_{f}-\beta_{b}\right) \\
\mathbf{J}_{f b}(\mathbf{r}, t)=-\left(\mu_{b f}^{s}+\mu_{b b}^{s}\right) \nabla \mathbf{u}_{f}^{s}-\left(\mu_{b f}^{0}+\mu_{b b}^{0}\right) \nabla \cdot \mathbf{u}_{f} \mathbf{U}, \quad \mathbf{j}_{f b}(\mathbf{r}, t)=-\sigma \mathbf{u}_{f} .
\end{gathered}
$$

The scalar transport coefficients are obtained by convolution of the tensor coefficients:

$$
\mu_{i f}^{s}=\frac{\beta_{f}}{5} \int_{t_{0}}^{t} d t_{1} \int d \mathbf{r}^{\prime}\left\langle\hat{\mathbf{J}}_{i}^{\prime s}(\mathbf{r}): S_{\left(t-t_{1}\right)}^{(N)} \hat{\mathbf{J}}_{2 f}^{\prime s}\left(\mathbf{r}^{\prime}\right)\right\rangle, \quad \mu_{i b}^{s}=\frac{\beta_{f}}{5} \int_{t_{0}}^{t} d t_{1} \int d \mathbf{r}^{\prime}\left\langle\hat{\mathbf{J}}_{i}^{\prime s}(\mathbf{r}): S_{\left(t-t_{1}\right)}^{(N)} \hat{\mathbf{J}}_{f b}^{\prime s}\left(\mathbf{r}^{\prime}\right)\right\rangle
$$




$$
\begin{gathered}
\mu_{b}^{s}=\frac{\beta_{b}}{5} \int_{t_{0}}^{t} d t_{1} \int d \mathbf{r}^{\prime}\left\langle\hat{\mathbf{J}}_{2 b}^{\prime s}(\mathbf{r}): S_{\left(t-t_{1}\right)}^{(N)} \hat{\mathbf{J}}_{f b}^{\prime s}\left(\mathbf{r}^{\prime}\right)\right\rangle \\
\mu_{i f}^{0}=\frac{\beta_{f}}{9} \int_{t_{0}}^{t} d t_{1} \int d \mathbf{r}^{\prime}\left\langle\hat{\mathbf{J}}_{i}^{\prime}(\mathbf{r}): S_{\left(t-t_{1}\right)}^{(N)} \hat{\mathbf{J}}_{2 f}^{\prime 0}\left(\mathbf{r}^{\prime}\right)\right\rangle, \quad \mu_{i b}^{0}=\frac{\beta_{f}}{9} \int_{t_{0}}^{t} d t_{1} \int d \mathbf{r}^{\prime}\left\langle\hat{\mathbf{J}}_{i}^{\prime}(\mathbf{r}): S_{\left(t-t_{1}\right)}^{(N)} \hat{\mathbf{J}}_{f b}^{\prime 0}\left(\mathbf{r}^{\prime}\right)\right\rangle \\
\mu_{b}^{0}=\frac{\beta_{b}}{9} \int_{t_{0}}^{t} d t_{1} \int d \mathbf{r}^{\prime}\left\langle\hat{\mathbf{J}}_{2 b}^{\prime}(\mathbf{r}): S_{\left(t-t_{1}\right)}^{(N)} \hat{\mathbf{J}}_{f b}^{\prime 0}\left(\mathbf{r}^{\prime}\right)\right\rangle \\
\lambda_{f}=\frac{1}{3} \int_{t_{0}}^{t} d t_{1} \int d \mathbf{r}^{\prime}\left\langle\hat{\mathbf{J}}_{3 f}^{\prime}(\mathbf{r}) \cdot S_{\left(t-t_{1}\right)}^{(N)} \Delta\left(\hat{\mathbf{J}}_{3 f}^{\prime}\left(\mathbf{r}^{\prime}\right)+\hat{\mathbf{p}}_{f}^{\prime}\left(\mathbf{r}^{\prime}\right) \frac{\beta_{f}\left(\mathbf{r}^{\prime}\right)}{m_{f} n_{f}\left(\mathbf{r}^{\prime}\right)}\left(\frac{\partial p_{f}}{\partial \beta_{f}}\right)_{\nu_{f}}\right)\right\rangle_{0} \\
\lambda_{b}=\frac{1}{3} \int_{t_{0}}^{t} d t_{1} \int d \mathbf{r}^{\prime}\left\langle\hat{\mathbf{J}}_{3 f}^{\prime}(\mathbf{r}) \cdot S_{\left(t-t_{1}\right)}^{(N)} \Delta \hat{\mathbf{J}}_{3 b}^{\prime}\left(\mathbf{r}^{\prime}\right)\right\rangle_{0} \\
\sigma=\frac{1}{6}\left(\beta_{f}+\beta_{b}\right) \int_{t_{0}}^{t} d t_{1} \int d \mathbf{r}^{\prime}\left\langle\hat{\mathbf{j}}_{f b}(\mathbf{r}) \cdot S_{\left(t-t_{1}\right)}^{(N)} \hat{\mathbf{j}}_{2 f}\left(\mathbf{r}^{\prime}\right)\right\rangle_{0}
\end{gathered}
$$

\section{Conclusion}

The technique developed in this paper to describe transport processes in confined geometries allows one to derive closed transport equations based on first principles. It is important that the characteristics in these equations, such as the stress tensor or heat flux vector, are expressed in terms of the parameters of the fluid-fluid and fluid-surface interaction forces. Therefore, specifying real material parameters, we can predict how the processes of momentum and energy transfer will occur in a particular microsystem. In this sense, the constructed equations are fairly universal.

The main conclusion is that transport processes in fluids under confined conditions differ significantly from fluid transport processes in bulk. Therefore, the viscosity and thermal conductivity coefficients of fluids in bulk and in nanochannels will be different. It is impossible to use the Green - Kubo formula to simulate the transport coefficients of fluids under confined conditions (using, for example, molecular dynamics method). In this case the shear viscosity coefficient is given by the formula:

$$
\mu=\mu_{f f}^{s}+\mu_{f b}^{s}+\mu_{b}^{s} .
$$

The first two terms are typical of a two-fluid medium. The last term does not have a classical analog. As a rule, we can neglect the space nonlocality of the relations (16), i.e. the transport relaxation kernels are $\delta$-functions of the coordinates $\mathbf{r}$ and $\mathbf{r}^{\prime}$. In this case, the viscosity coefficients are determined by the following formulas:

$$
\mu_{f f}^{s}=\frac{\beta_{f}}{5} \int_{t_{0}}^{t} d t_{1}\left\langle\hat{\mathbf{J}}_{i}^{\prime s}(\mathbf{r}): S_{\left(t-t_{1}\right)}^{(N)} \hat{\mathbf{J}}_{2 f}^{\prime s}(\mathbf{r})\right\rangle, \quad \mu_{f b}^{s}=\frac{\beta_{f}}{5} \int_{t_{0}}^{t} d t_{1}\left\langle\hat{\mathbf{J}}_{i}^{\prime s}(\mathbf{r}): S_{\left(t-t_{1}\right)}^{(N)} \hat{\mathbf{J}}_{f b}^{\prime s}(\mathbf{r})\right\rangle
$$




$$
\mu_{b}^{s}=\frac{\beta_{b}}{5} \int_{t_{0}}^{t} d t_{1}\left\langle\hat{\mathbf{J}}_{2 b}^{\prime s}(\mathbf{r}): S_{\left(t-t_{1}\right)}^{(N)} \hat{\mathbf{J}}_{f b}^{\prime s}(\mathbf{r})\right\rangle .
$$

The thermal conductivity process is determined by the gradients of the fluid and wall temperature (see Eq. (15)). If the channel walls have the same temperature as the fluid, the thermal conductivity coefficient of the fluid under confined conditions is described by the formula:

$$
\lambda=\lambda_{f}+\lambda_{b},
$$

where, in local approximation, the coefficients have the following form:

$$
\begin{gathered}
\lambda_{f}=\frac{1}{3} \int_{t_{0}}^{t} d t_{1}\left\langle\hat{\mathbf{J}}_{3 f}^{\prime}(\mathbf{r}) \cdot S_{\left(t-t_{1}\right)}^{(N)} \Delta\left(\hat{\mathbf{J}}_{3 f}^{\prime}(\mathbf{r})+\hat{\mathbf{p}}_{f}^{\prime}(\mathbf{r}) \frac{\beta_{f}(\mathbf{r})}{m_{f} n_{f}(\mathbf{r})}\left(\frac{\partial p_{f}}{\partial \beta_{f}}\right)_{\nu_{f}}\right)\right\rangle_{0}, \\
\lambda_{b}=\frac{1}{3} \int_{t_{0}}^{t} d t_{1}\left\langle\hat{\mathbf{J}}_{3 f}^{\prime}(\mathbf{r}) \cdot S_{\left(t-t_{1}\right)}^{(N)} \Delta \hat{\mathbf{J}}_{3 b}^{\prime}\left(\mathbf{r}^{\prime}\right)\right\rangle_{0} .
\end{gathered}
$$

Equations $(17)-(20)$ are the generalization of the known Green - Kubo relations. The formula for transport coefficients of fluids under confined conditions is rather complex. We can hope to derive simple relations only for rarefied gases. In this case, the gas evolution is described by the Boltzmann's equation. There is a single method for calculating the transport coefficients of dense gases or liquids. This is the molecular dynamics (MD) method. In this case, formulas (18), (20) should be used. However, in some MD simulations of nanoflows, attempts are made to use conventional fluid-dynamic equation and Green - Kubo formulas for transport coefficients. Clearly, this approach is inappropriate for such small systems.

Note, finally, that the possible elasticity and compliance of the walls can be taken into account using the formalism developed. For this, however, a different quasi-equilibrium ensemble is required.

\section{Acknowledgement} 19-00312).

This work was supported in part by the Russian Scientific Foundation (Grant No. 14-

\section{References}

[1] Andryushchenko V., Rudyak V. Self-diffusion coefficient of molecular fluid in porous media. Defect and Diffusion Forum, 2011, 312-315, P. 417-422.

[2] Rudyak V.Ya., Belkin A.A., Egorov V.V., Ivanov D.A. About fluids structure in microchannels. Int. J. of Multiphysics, 2011, 5 (2), P. 145-155.

[3] Judy J., Maynes D., Webb B.W. Characterization offrictional pressure drop for liquid flows through microchannels. Int. J. Heat Mass Transfer, 2002, bf 45, P. 3477-3489.

[4] Liu Y., Wang Q. Transport behavior of water confined in carbon nanotubes. Phys. Rev. B, 2005, 72, P. 085420.

[5] Hanasaki I., Nakatani A. Hydrogen bond dynamics and microscopic structure of confined water inside carbon nanotubes. J. Chem. Phys., 2006, 124, P. 174714.

[6] Karniadakis G., Beskok A., Aluru N. Microflows and nanoflows: Fundamentals and Simulation. Springer, 2005, 824 p.

[7] Rudyak V.Ya., Belkin A.A., Egorov V.V., Ivanov D.A. Modeling fluid flows in nanochannels by molecular dynamics method. Nanosystems: Physics, Chemistry, Mathematics, 2011, 2 (4), P. 100-112. 
[8] Kharlamov G.V., Rudyak V.Ya. The equilibrium fluctuations in small open systems. Physica A, 2004, 340, P. 257-264.

[9] Pfahler J., Harley J., Bau H., Zemel J.N. Gas and liquid flow in small channels. Micromech. Sensors, Actuators, Syst., 1991, 32, P. 49-58.

[10] Kobryn A.E., Kovalenko A. Molecular theory of hydrodynamic boundary conditions in nanofluidics. J. Chem. Phys., 2008, 129, P. 134701.

[11] Rudyak V.Ya. Statistical aerohydromechanics of homogeneous and heterogeneous media. V.2. Hydromechanics. NSUACE, Novosibirsk, 2005.

[12] Rudyak V.Ya., Belkin A.A. Statistical hydromechanics of multiphase systems. J. Aerosol Sci., 1994, 25, P. 387-388.

[13] Rudyak V.Ya., Belkin A.A. Equations of multifluid hydrodynamics. Mathematical Modeling, 1996, 8, P. 3337.

[14] Rudyak V.Ya., Belkin A.A. Equations of multifluid hydrodynamics for heterogeneous systems with rotational degrees of freedom. Siberian J. of Industrial Mathematics, 2002, 1 (9), P. 145-156.

[15] Zubarev D.N. Nonequilibrium statistical thermodynamics. Consultants Bureau, New York, 1974.

[16] Rudyak V.Ya. Statistical theory of dissipative processes in gases and liquids. Nauka, Novosibirsk, 1987. 